\title{
The Effect of Low-Amplitude Oscillations of the Load on the Local Stiffness of Materials in Loaded Nanocontacts Detected by Methods of Dynamic Nanoindentation
}

\author{
Yu.I. Golovin ${ }^{1,2 *}$, V.V. Korenkov ${ }^{1}$, S.S. Razlivalova ${ }^{1}$ \\ ${ }^{1}$ Scientific Research Institute of Nanotechnologies and Nanomaterials, \\ G.R. Derzhavin Tambov State University, 33, Internasionalnaya St., Tambov, 392000, Russia; \\ ${ }^{2}$ Lomonosov Moscow State University, GSP-1, Leninskie Gory, 1, Bld. 11, Moscow, 119991, Russia \\ * Corresponding author: Tel.: 8(4752) 5326 80.E-mail: yugolovin@yandex.ru
}

\begin{abstract}
The role of low-amplitude oscillations in the formation of mechanical properties of nanocontacts with different values of the ratio of hardness $H$ to the elastic modulus $E$ was studied by dynamic nanoindentation (DNI). Various methods for processing raw data and extracting nanocontact characteristics from them have different sensitivity to low-amplitude oscillations of the load. It is found that under certain test conditions, the addition of low-amplitude oscillations does not affect the mechanisms, kinetics and magnitude of the plastic deformation of the material under the indenter. The threshold values of the oscillation amplitude for materials to soften locally in the nanocontact interaction are determined.
\end{abstract}

\section{Keywords}

Nanocontact harmonic stiffness; dynamic nanoindentation; low-amplitude oscillations; acoustoplastic effect.

(C) Yu.I. Golovin, V.V. Korenkov, S.S. Razlivalova, 2018

\section{Introduction}

Due to the complexity of the physicochemical processes occurring in dynamic nanocontacts during dry friction, wear, grinding, etc., the regularities and atomic deformation mechanisms under these conditions remain unclear. The desire to increase the amount of information about the behavior of the material in dynamic nanocontacts and thin near-surface layers initiated the invention and the subsequent development of an innovative instrumental option of nanoindentation (NI) - continuous stiffness measurement (CSM). The principle of the CSM mode is to superimpose lowamplitude harmonic perturbation $F_{o s c}=F_{a} \sin \omega t$, on the initial slowly growing load $F(t)$, where $F_{a}$ is amplitude of additional harmonic force, $\omega$ is its cyclic frequency (Fig. 1). Sometimes this method is called dynamic nanoindentation (DNI) and is realized with both prismatic and spherical indenters [1-5]. The CSM option allows one to study mechanical characteristics in films with a thickness from one nanometer to many microns, in separate phases of composite materials, multilayer coatings and macrohomogeneous samples, when the size of the locally deformed region changes by 4-5 orders of magnitude (the volume of material involved into deformation increases up to 15 orders of magnitude!) [6].

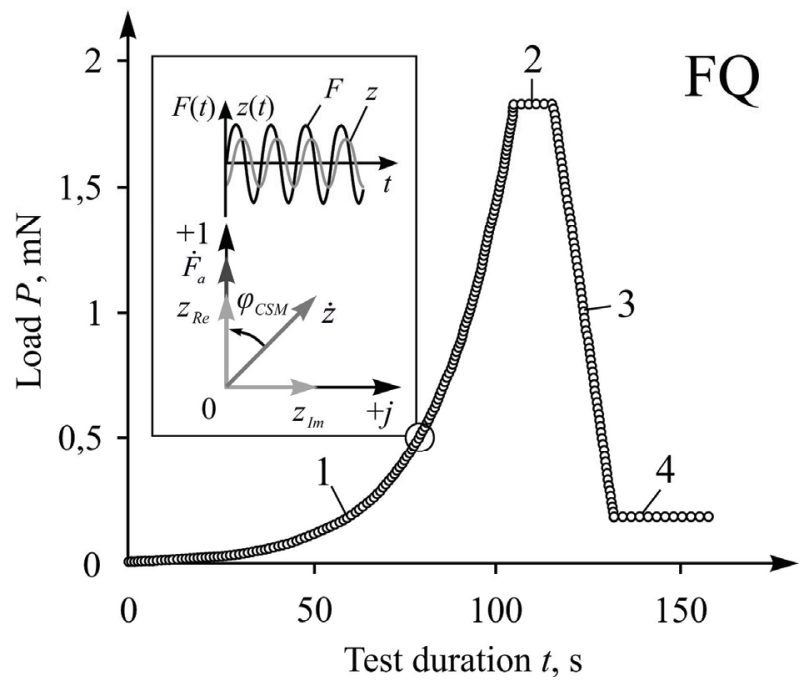

Fig. 1. Typical load profile used in the work: 1 - loading curve; 2 - constant load; 3 - unloading; 4 - temperature drift detection (On the inset - the principle of the CSM mode. Here $Z_{R e}$ and $Z_{I m}$ are the real and imaginary parts of displacement oscillations) 
The CSM makes it possible to study indentation size effects (ISE) during a single test cycle [7], to conduct hundreds of tests on the resistance of plastic deformation and fatigue in a nanoscale on one sample to collect reliable statistics, to measure wear resistance of individual parts of micro- and nanomechanical systems (MEMS / NEMS), to investigate the viscoelastic properties of biomolecular objects (for example, healthy and cancer cells), atomic mechanisms of constrained local deformation [8-10] and much more. Thus, at the scale, the CSM occupies an intermediate position between the AFM methods, with the size of the test region $\sim 1 \mathrm{~nm}$, and the macroscopic methods of dynamic mechanical analysis (DMA) and internal friction (IF), but in nature and in the principle of implementation is a local analogue of the latter [11-13].

Earlier, we found the existence of some critical amplitude of load / deformation oscillations, at which the atomic structure of the material does not undergo additional irreversible changes and practically does not dissipate the elastic energy during the test [14]. Such "non-destructive" tests are reliable only up to a certain critical value of the amplitude of the oscillations. In the supercritical modes, an irreversible rearrangement of the initial structure begins (for example, the multiplication of dislocations, their movement over large distances, changes in the structure of subgrain and grain boundaries, etc.). It is known that simultaneous action of a relatively slowly increasing load and an additional supercritical oscillatory force causes the acoustoplastic effect (APE), expressed in decreasing the yield strength, the strain hardening coefficient, etc. [15-17]. Analogous phenomena can also be expected in dynamic nanoindentation.

Along with the effect of the harmonic load on the defect structure of the material, the methodological errors of the CSM method [3-5, 18, 19] could be mentioned as a possible cause of the usually observed softening at the contact spot under the influence of oscillations. However, the amount of available data on atomic mechanisms and the impact of oscillations on the behavior of dynamic contacts are still insufficient, so that reliability and consistency of DNI results are questionable. For example, Cordill et al. [20] suggested that the periodic action of low-frequency lowamplitude oscillations can cause material fatigue in the nanocontact, that is, modify the mechanical properties of the sample under study in the contact spot. This hypothesis has been experimentally confirmed $[8,21]$, however, only at much larger oscillation amplitudes than is commonly used in nanoindentation. Another problem of DNI is the observed discrepancy between dynamic and quasi-statistic tests at very small indentation depths $[22,23]$. Later they were taken into account, and it was shown in [19] that for materials with a Young's modulus to hardness ratio $(E / H)>10$, the assumption of a unloading linearity is not fully satisfied for oscillations whose amplitude is more than a few nanometers, which leads to systematic overestimation of the stiffness.

The identified problems allow us to formulate the goals and objectives of this study as follows:

a) finding the range of loads / deformations at which the CSM method does not affect the mechanisms, kinetics and magnitude of localized plastic deformation under the indenter, for materials having a Young's modulus to hardness ratio $(E / H)>10$;

b) determining quantitative regularities of the discrepancy between dynamic measurements and quasistatistical tests at very small indentation depths in materials with various $E / H$ ratios.

\section{Equipment, materials and preparation of samples}

The experimental part of the work was performed using Agilent's NanoIndenter ${ }^{\circledR}$ G200 instrument in the load range of $0.01-100 \mathrm{mN}$ using a Berkovich diamond indenter having tip radius $R=20 \mathrm{~nm}$. The testing was carried out by two main loading modes: a) traditional quasi-static without adding oscillations; b) using the CSM method with superposition of an additional oscillation of the indenter displacement in the direction perpendicular to the test surface, with amplitude $z=0.5-8 \mathrm{~nm}$ and frequency $10-45 \mathrm{~Hz}$. During all the experiments, the rate of relative quasistatic deformation was kept constant $\dot{\varepsilon}=0.05 \mathrm{~s}^{-1}$ by means of feedback circuits in the instrument, which made it possible to exclude the effect of the loading rate factor on the hardness value. The maximum depth of prints in the control samples (for which $z=0$ ), preset in the test report was $25,50,100,200,500,1000,1500$ and $2000 \mathrm{~nm}$. All prints were located at a distance of at least $50 \mu \mathrm{m}$ from each other in order to avoid their mutual influence. The hardness values of $H$ and the Young's modulus $E$ were averaged from the data of ten prints.

As research objects, several representatives of different classes of materials were selected, which differ in the magnitude of the ratio $E / H$ :

a) high-purity $(0.9999 \mathrm{wt} \%)$ polycrystalline (average grain size $\sim 100 \mu \mathrm{m})$ soft and plastic $(E / H=$ = 240) metal with face-centered cubic (fcc) lattice (Al);

b) hard metal with body-centered cubic lattice (bcc) $\mathrm{W}(E / H=70)$;

c) rigid semiconductor $\mathrm{GaAs}(E / H=14)$.

The surface of polycrystalline Al samples with dimensions $10 \times 10 \times 3 \mathrm{~mm}^{3}$ was prepared by mechanical grinding with $\mathrm{SiC}$ abrasive (1200 grit) and polishing with diamond paste on the Buehler Vector 
Power Grid device for sample preparation. After mechanical polishing, the surface layer was electrochemically polished for 3 minutes at a current density $1 \mathrm{~A} / \mathrm{cm}^{2}$ in $10 \% \mathrm{HCl}$ solution in ethanol to eliminate the near-surface deformation-hardened layer.

Non-doped samples of single-crystal GaAs (99.999 \%, Sigma Aldrich) with orientation (001) were cut in the form of a parallelepiped of $10 \times 5 \times 1 \mathrm{~mm}^{3}$ and were subsequently polished with $\mathrm{Al}_{2} \mathrm{O}_{3}$ powder with a particle size of 5,1 and $0.3 \mu \mathrm{m}$.

Polycrystalline samples of tungsten $\left(6 \times 12 \times 3 \mathrm{~mm}^{3}\right.$; $99.97 \%$ purity, average grain size 30 microns) were made by melting a large ingot in an inert gas atmosphere. Samples were also polished in a standard manner using the Buehler Vector Power Grid to a mirror finish. The final surface roughness of the samples was measured by the AFM method in the "tapping mode" at a site of $4 \mu^{2}$. The root mean square roughness value for all samples did not exceed $2 \mathrm{~nm}$.

The basic nanocontact characteristics - hardness $H$ and Young's modulus $E$ were calculated in four different ways: 1) by the Oliver-Pharr Method (OPM) $[1,2,24] ; 2)$ by the CSM method $[1-7,25]$; 3) by the OPM method $[1,2,25]$ from the analysis of the unloading branch of the $P$ - $h$ diagram near $P_{\max }$ and 4) by direct measurement of the area of the projection $A_{c}$ of the print in an atomic force microscope [26]. In greater detail, the method for calculating the nanocontact characteristics is given in [14].

\section{Experimental results}

Materials with a high ratio $(E / H)$ are characterized by a strong deviation from the linearity of the unloading branch of the loading diagram $[3,4,7,19$, $24,25]$. For most ceramics and metals, the value of $E / H$ varies between 10-300. In accordance with this, the shape of the unloading curve also changes significantly. Experimentally the value of harmonic contact stiffness $S_{H}$ and harmonic contact damping $D_{i}$ of the test material can be calculated from the analysis of the dynamic response of the system:

$$
1 / S_{H}=1 /\left(\left(K_{i}-m \omega^{2}\right)^{2}+\left(D_{i} \omega\right)^{2}\right)^{0,5},
$$

where $K_{i}$ is suspension spring stiffness, $m$ is weight of the moving parts of the measuring head, $\omega$ is oscillation circular frequency. The value of the elastic and damping constants of the device is known: $K_{i}=92.02 \mathrm{~N} / \mathrm{m}, m_{i}=11.6 \mathrm{~g}, D_{i}=2.66 \mathrm{~N} \cdot \mathrm{s} / \mathrm{m}$, which allows finding the values of $H$ and $E$ by standard OPM $[1,2,25]$. In the paper, the dependences of both the standard stiffness $S=d P / d h$ in the absence of oscillations (here $P$ is the magnitude of the load on the indenter, $h$ is the depth of indenter introduction) in the nacontact, and harmonic stiffness $S_{H}$ on the ratio $E / H$ have been studied.

As follows from the graphs of the dependence of the harmonic stiffness $S_{H}$ in the contact of the indenter with the sample on the indenter penetration depth $h$ for different amplitudes of the additional oscillations $z$ shown in Fig. 2, the largest value of $S_{H}$ has a tungsten refractory metal with a bcc lattice with $E / H=70$. The minimum $S_{H}$ of the tested materials has fcc aluminum metal for which $E / H=240$.

For harmonic oscillations in $\mathrm{Al}$, it can be noted that stiffness has the greatest value for each of the materials studied in the absence of oscillations, and harmonic oscillations of displacement above some critical amplitude cause softening of the material in the dynamic nanocontact. Al is the only material in which the dependence of the harmonic stiffness on the amplitude of the additional oscillations $z$ becomes observable, which coincides with the known literature data $[23,27]$. For $\mathrm{W}$ and GaAs, the amplitude of the additional stiffness oscillations lies within the measurement uncertainty.

According to the literature data for the spherical indenter [9], it is known that the measured contact stiffness depends strongly not only on the amplitude, but also on the frequency of the additional oscillations used in the experiment. Fig. 3 shows the dependence of the harmonic stiffness on the oscillation frequency in the materials under investigation for the sharp Berkovich indenter for fixed oscillation amplitude $z=2 \mathrm{~nm}$. As could be seen from this figure, the lower the frequency of the harmonic oscillations, the less is

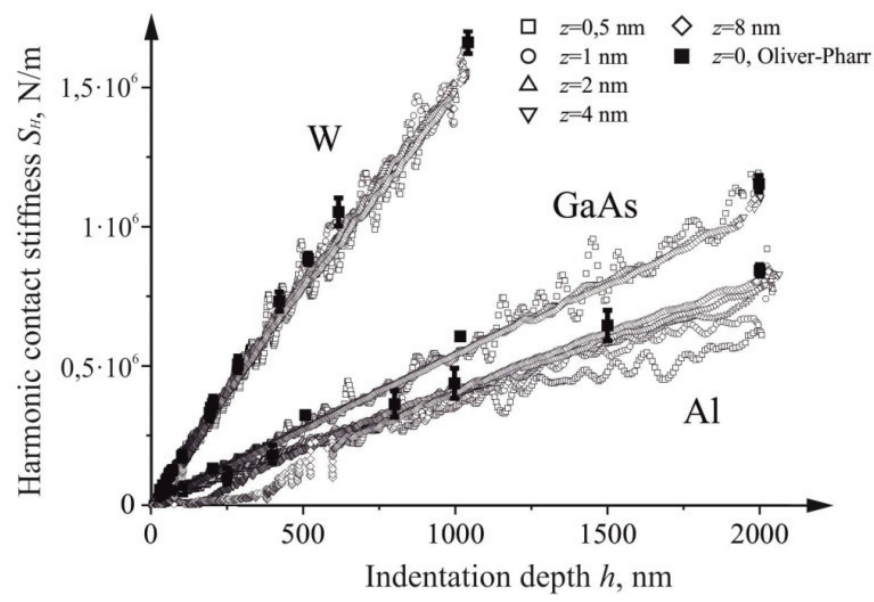

Fig. 2. Correlation of harmonic stiffness in the contact of the indenter with the sample and the depth of indentation for various amplitudes of harmonic displacement 


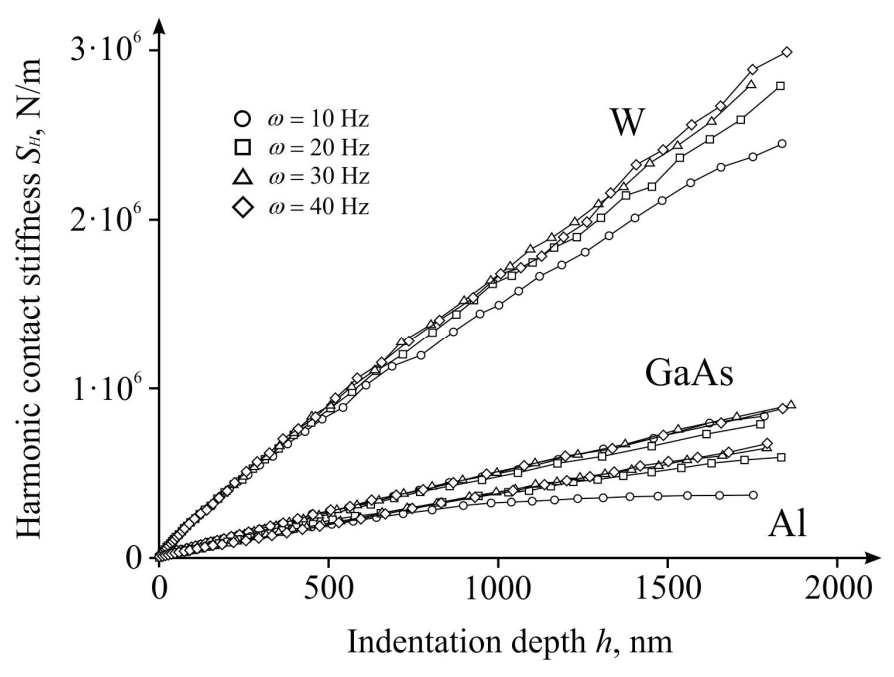

Fig. 3. Correlation of contact stiffness and the depth of indentation for various frequencies of harmonic oscillations and amplitude of oscillations $z=2 \mathrm{~nm}$

the contact stiffness in the investigated frequency range.

It can also be stated that the smallest angle with the stiffness axis is the family of curves $S_{H}(\omega)$ for W, the largest one is for $\mathrm{Al}$, and $\mathrm{GaAs}$ occupies an intermediate position between them. It is not yet possible to explain why, for a metal with a bcc lattice, the harmonic stiffness curves with respect to frequency are separated most clearly and much better than for fcc metals. For all materials, the following general rule is fulfilled - the increase in rigidity occurs linearly with increasing $h$ to a certain critical value, starting from which the stiffening growth curves diverge. For $\mathrm{W}$ this difference is more pronounced than for $\mathrm{Al}$, whereas for $\mathrm{GaAs}$ it does not go beyond the measurement error.

As was established in our work [14] earlier, the value of the main load $P$, necessary for imprinting the indenter to a fixed depth, consistently decreased with increasing amplitude of displacement oscillations. Hence, for a fixed load the superposition of additional oscillations should lead to an increase of indentation depth $h$ with increasing $z$. As a measure of the effect of superimposed oscillations on the local deformation under the indenter, it was also proposed to take the depth difference $\delta h$ between the loading curves with the addition of additional oscillation of the displacement $(z>0)$, and without it $(z=0)$ at each point with the same load P. This allows us to take the value of $\delta h$ as a quantitative characteristic of the additional deformation under the influence of oscillations. The observed values of additional deformation $\delta h$ in the presence of additional harmonic oscillation grow with increasing $E / H$ (Fig. 4).

We can note a small value of $\delta h$ at the initial stage of indentation. Perhaps this is a consequence of the fact that at the initial stage of the interaction of the indenter

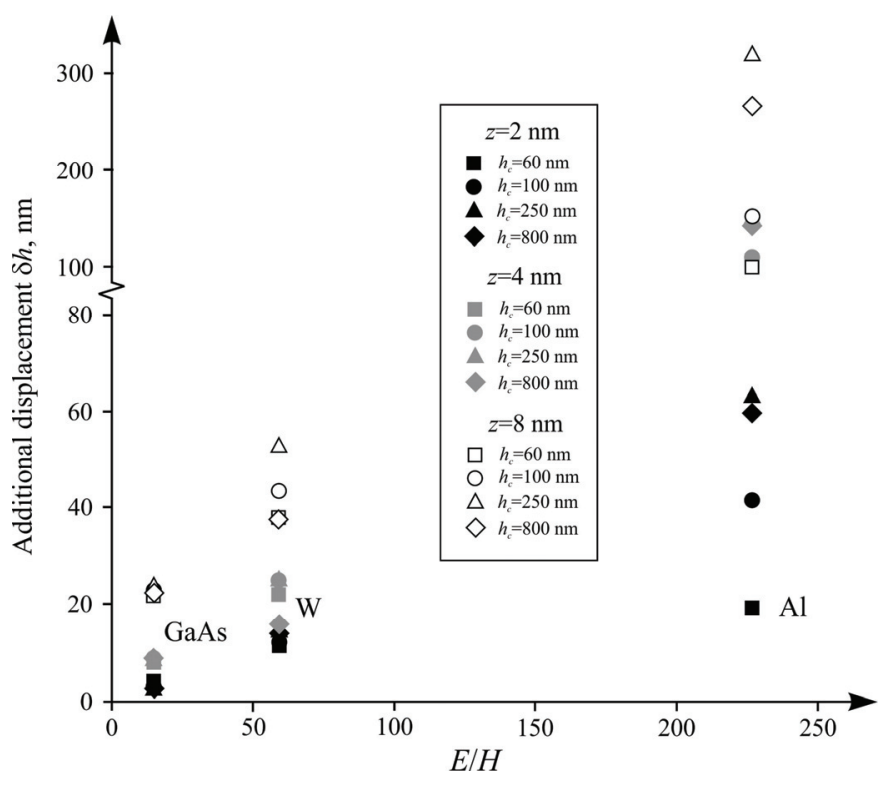

Fig. 4. The value of the additional deformation under the influence of the oscillating load at different penetration depths of the indenter for materials with different value of the ratio $E / H$

with the surface of the sample, the response of the system for determining the moment of contact and the zero point for indentation depth recording can be added to the response of the material. A sudden change in stiffness in the case of a short-term contact of oscillating indenter with the sample surface should lead to the activation of feedback loops and an increase in the amplitude of the oscillations, and the discontinuity of the contact to the inverse process. These transient processes can increase the error in determining the position of the surface. However, after the establishing steady (uninterrupted) contact, the role of this factor should decrease with increasing $h$.

Analysis of the ratio of the change in hardness $\Delta H / H$ when the indenter is loaded with the addition of oscillation of the displacement to the hardness determined by the OPM method without imposing oscillation, on the value of the ratio $E / H$ for indenting a single crystal of gallium arsenide and polycrystals $\mathrm{W}$ and $\mathrm{Al}$ indicates that the quantity $\Delta H / H<0$ under any conditions (Fig. 5).

Finally, for each material studied in this paper, the limiting amplitude of the critical vibrations $z_{c}$ (i.e., the amplitude of the harmonic oscillations, not causing a noticeable effect on the microstructure of the material) was determined. Fig. 6 shows the dependence of $z_{c}$ on the ratio $E / H$. The value of $z_{c}$ can reach $3.5 \mathrm{~nm}$ for GaAs single crystals, whereas for the W polycrystals the critical amplitude never exceeds $3 \mathrm{~nm}$. The observed amplitude of critical oscillations for $\mathrm{Al}$ is $2 \mathrm{~nm}$, which is close to the critical threshold $z_{c}{ }^{*}$ 


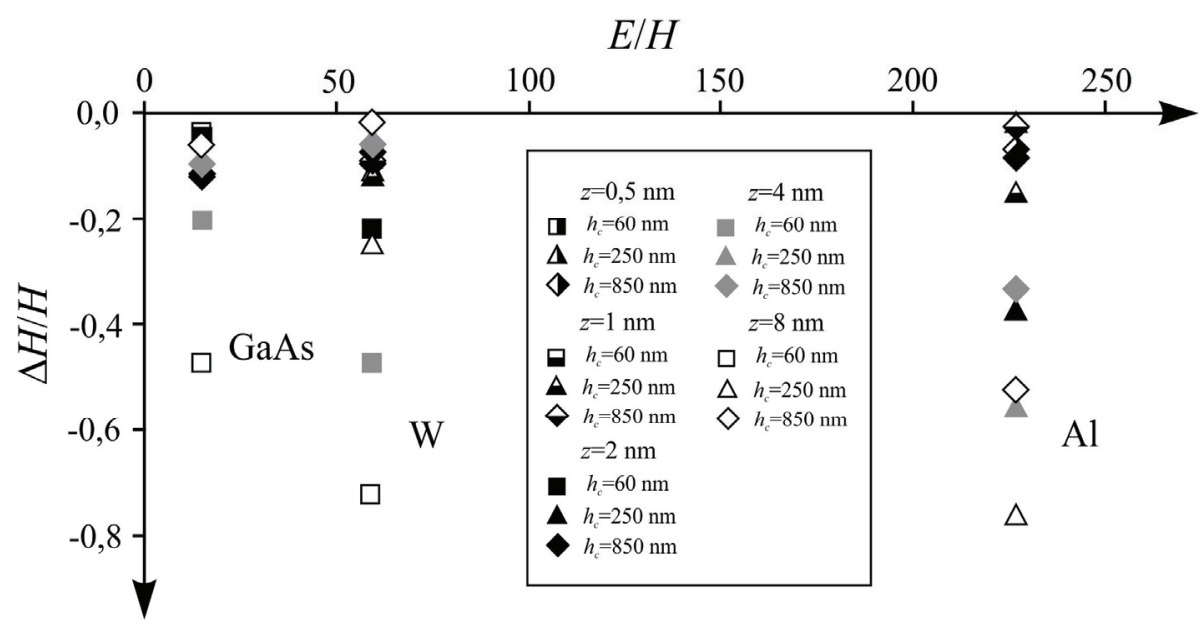

Fig. 5. Dependence of the relative hardness $\Delta H / H$ for materials with different values of $E / H$ for different values of the contact depth $h_{c}$ and the oscillation amplitude in the range $0.5-8 \mathrm{~nm}$

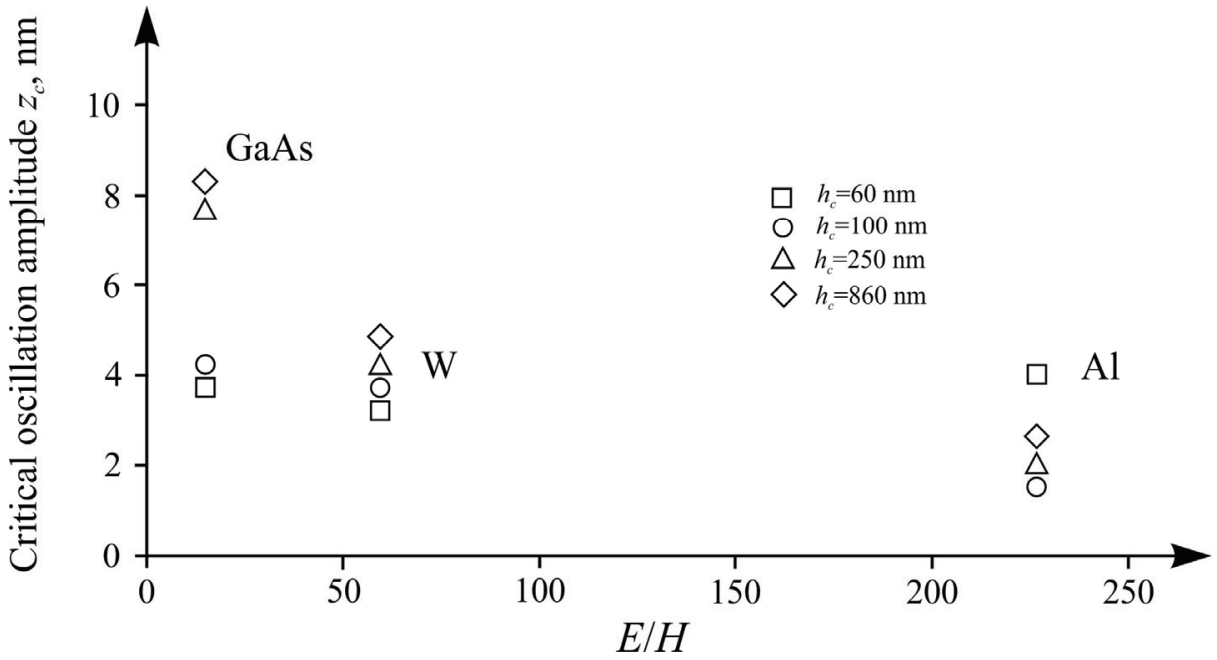

Fig. 6. Dependence of the critical amplitude of harmonic oscillations $z_{c}$ on the value of the ratio $E / H$ of different materials for different values of the depth of the nanocontact

recommended for CSM measurements in a mode free from systematic hardness overestimation for both spherical and prismatic indenter [18, 19]. To explain this behavior of the material, it can be assumed that the cause of softening is the fatigue of the material in the nanocontact under the action of a large number of cycles of alternating loading [8]. Experimental studies of fatigue in nanocontacts can help to determine the validity of this hypothesis, but they have not been carried out to date.

\section{Conclusion}

The studies carried out for materials with different Young's modulus ratio to hardness $(E / H)$ made it possible to establish the following.

1. The harmonic stiffness $S_{H}$ of the polycrystalline bcc tungsten metal in the contact of the indenter with the sample proves to be higher than that of the soft and plastic fcc metal Al.

2. It has been found that the softer and more pliable the material is, the more noticeable is the effect of harmonic oscillations of the loading force.

3. The quantitative dependence of the harmonic stiffness on the frequency of oscillations in the materials studied has been revealed. In the frequency range $10-40 \mathrm{~Hz}$, the following rule is fulfilled - the higher the oscillation frequency is, the stronger is its effect on the harmonic stiffness of the nanocontact.

4. For materials with different $E / H$ values, it is shown that the greater the ratio of the modulus of elasticity to hardness is, the greater is the value of the measured additional deformation caused by the oscillations.

5. It has been found that the amplitude of the critical oscillations for GaAs is $3.5 \mathrm{~nm}$, while for $\mathrm{W}$ this value is $3 \mathrm{~nm}$, and for $\mathrm{Al} z_{c}=2 \mathrm{~nm}$. 


\section{Acknowledgements}

The work was carried out as part of the Government task for scientific research 16.2100.2017 / IF: Analysis of the influence of nanometer-scale oscillations of displacement on the physical and mechanical properties of materials; as well as the grant of the Russian Science Foundation, project No. 16-19-10405: Analysis and modeling of local deformation.

\section{References}

1. Oliver W.C., Pharr G.M. Measurement of hardness and elastic modulus by instrumented indentation: Advances in understanding and refinements to methodology. J. Mater. Res., 2004, vol. 19, pp. 3-20.

2. Oliver W.C., Pharr G.M. An improved technique for determining hardness and elastic modulus using load and displacement sensing indentation experiments. J. Mater. Res., 1992, vol. 7, pp. 1564-1583.

3. Hay J., Agee P., Herbert E. Continuous stiffness measurement during instrumented indentation testing. Experimental Techniques, 2010, vol. 5-6, pp. 86-94.

4. Li X., Bhushan B. A review of nanoindentation continuous stiffness measurement technique and its applications. Materials Characterization, 2002, vol. 48, pp. 11-36.

5. Pathak S., Kalidindi S.R. Spherical nanoindentation stress-strain curves. Materials Science and Engineering $R$, 2015, vol. 91, pp. 1-36.

6. Hayes S.A., Goruppa A.A., Jones F.R. Dynamic nanoindentation as a tool for the examination of polymeric materials. J. Mater. Res., 2004, vol. 19, pp. 3298-3306.

7. Durst K., Maier V. Dynamic nanoindentation testing for studying thermally activated processes from single to nanocrystalline metals. Curr. Opin. Sol. State Mater. Sci., 2015, vol. 19, pp. 340-353.

8. Golovin Yu.I., Ivolgin V.I., Korenkov V.V., Farber B.Y. The Hardening and Loss of Strength of Thin Surface Layers of LiF Monocrystals and Zirconium Ceramics under Conditions of Cyclic Nanoindenting. Physics of the Solid State, 2001, vol. 43, no. 10, pp. 1917-1922.

9. Schuh C.A., Masonanda J.K., Lund C. Quantitative insight into dislocation nucleation from high-temperature nanoindentation experiments. Nature Materials, 2005, vol. 4, pp. 617-621.

10. Liang H.Y., Woo C.H., Huang H., Ngan A.H.W., $\mathrm{Yu}$ T.X. Dislocation nucleation in the initial stage during nanoindentation. Phil. Mag., 2003, vol. 83, pp. 3609-3622.

11. Foschia R., Jobin M., Hengsberger S. Local dynamic mechanical analysis. Micron, 2009, vol. 40, pp. 51-55.

12. Trunov M.L., Bilanich V.S., Dub S.N. Nanoindentation Study of the Time-Dependent Mechanical Behavior of Materials. Tech. Phys., 2007, vol. 52, pp. 1298-1305.
13. Nowick A.S., Berry B.S. Anelastic relaxation in crystalline solids. New York, Academic Press, 1972.

14. Golovin Yu.I., Korenkov V.V., Razlivalova S.S. The effect of small-amplitude load oscillations on the nanocontact characteristics of materials in nanoindentations. Phys. Solid States, 2017, vol. 59, no. 6, pp. 1106-1117.

15. Tanibayashi M. Theory of the Blaha Effect. Phys. Stat. Sol. (a), 1991, vol. 128, pp. 83-94.

16. Siu K.W., Ngan A.H.W. Understanding acoustoplasticity through dislocation dynamics simulations. Phil. Mag., 2011, vol. 91, pp. 4367-4387.

17. Siu K.W., Ngan A.H.W. Oscillation-induced softening in copper and molybdenum from nano- to micro-length scales. Mater. Sci. Engineering, 2013, vol. A 572, pp. 56-64.

18. Vachhani S.J., Doherty R.D., Kalidindi S.R. Effect of Continuous Stiffness Measurement on the Mechanical Properties Extracted using Spherical Nanoindentation. Acta Mater., 2013, vol. 61, pp. 3744-3751.

19. Pharr G.M., Strader J.H., Oliver W.C. Critical Issues in Making Small-Depth Mechanical Property Measurements by Nanoindentation with Continuous Stiffness Measurement. J. Mater. Res., 2009, vol. 24, pp. 653-666.

20. Cordill M.J., Lund M.S., Parker J., Leighton C., Nair A.K., Farkas D., Moody N.R., Gerberich W.W. The nanojackhammer effect in probing near-surface mechanical properties. Inter. J. Plast., 2009, vol. 25, no. 11, pp. 2045-2058.

21. Siu K.W., Ngan A.H.W. The continuous stiffness measurement technique in nanoindentation intrinsically modifies the strength of the sample. Phil Mag., 2013, vol. 93, no 5, pp. 449-467.

22. Durst K., Backes B., Göken M. Indentation size effect in crystalline materials: correcting for the size of the plastic zone. Scripta Mater., 2005, vol. 52, pp. 1093-1097.

23. Merle B., Maier V., Göken M., Durst K. Experimental determination of the effective indenter shape and $\varepsilon$-factor for nanoindentation by continuously measuring the unloading stiffness. J. Mater. Res., 2012, vol. 27, no 1, pp. 214-221.

24. Golovin Yu.I. Nanoindentation and mechanical properties of solids in submicrovolumes, thin near-surface layers and films: Review. Phys. Solid State, 2008, vol. 50, pp. 2205-2236.

25. Golovin Yu.I. Nanoindentation and its capabilities. Mashinostroenie, Moscow, 2009. (Rus)

26. Charleux L., Keryvin V., Nivard M., Guin J.-P., Sangleboeuf J.-C., Yokoyama Y. A method for measuring the contact area in instrumented indentation testing by tip scanning probe microscopy imaging. Acta Mater., 2014, vol. 70 , pp. 249-258.

27. Merle B., Maier-Kiener V., Pharr G.M. Influence of modulus-to-hardness ratio and harmonic parameters on continuous stiffness measurement during nanoindentation. Acta Mater., 2017, vol. 134, pp. 167-176. 